\title{
The Packing of Helical and Zigzag Chains and Distribution of Interstitial Voids in Expanded Liquid Se near the Semi- conductor to Metal Transition
}

\author{
Kenji Maruyama ${ }^{1, *}$, Satoshi Hiroi(Sato ${ }^{2}$, Hirohisa Endo $^{3}$, Hideoki Hoshino ${ }^{4}$, Takashi \\ Odagaki ${ }^{5}$, and Friedrich Hensel $^{6}$ \\ ${ }^{1}$ Department of Chemistry, Faculty of Science, Niigata University, Niigata 50-2181, Japan \\ ${ }^{2}$ Toyota Technological Institute, Nagoya 468-8511, Japan \\ ${ }^{3}$ Department of Physics, Faculty of Science, Kyoto University, Kyoto 606-8224, Japan \\ ${ }^{4}$ Faculty of Education, Hirosaki University, Hirosaki 036-8560, Japan \\ ${ }^{5}$ Research Institute for Science Education, Kyoto 603-8346, Japan \\ ${ }^{6}$ Physikalische Chemie, Philipps-Universität Marburg, 35032 Marburg, Germany
}

\begin{abstract}
The reverse Monte Carlo (RMC) and Voronoi-Delaunay (VD) void analyses were applied to study the modification of chain geometries near the semiconductor (SC) to metal (M) transition in expanded liquid Se along the isochore of $d=3.4 \mathrm{~g} / \mathrm{cm}^{3}$.

Fluctuations of dihedral angles with increasing temperature and pressure cause modification of the helical $(\mathrm{H})$ chain to the planar zigzag $(\mathrm{Z})$ chain conformations. The distribution of voids size $\left(r_{V}\right)$ supported by chain segments and distances to the 4th $\sim 6$ th neighbor atoms on the chain segments provide information on the stacking of planar zigzag chains compensated by empty space (L-voids, $r_{V} \sim 3.6 \AA$ ) which leads to the formation of metallic domains. Near $\mathrm{SC}-\mathrm{M}$ transition region the number fraction $N_{Z} / N_{H}$ for $\mathrm{Z}$ and $\mathrm{H}$ chain segments increases.
\end{abstract}

\section{Introduction}

Expanded liquid (1-) Se transforms from semiconducting to metallic state near the critical point accompanying the volume contraction. The reverse Monte Carlo (RMC) simulation[1] and Voronoi-Delaunay (VD) void analyses[2,3] on the basis of structural data at various temperatures and pressures are useful tools to clarify the relationship between the packing of chains and voids in the intermediate scale near the SC-M transition.

\section{Results and Discussion}

X-ray scattering measurements have been performed with the white X-ray beamline BL28B2 at SPring-8. The monocrystalline saphire cell which has been developed by Tamura and Inui [4] at Hiroshima University was used to maintain the liquid Se as thin film. The apparatus containing the sample cell and electrinc furnace is described in Ref. [5]. The scattering intensity was obtained with the energy dispersion method. After the corrections for absorption,

\footnotetext{
*e-mail: maruken@sc.niigata-u.ac.jp
} 
incoherent scattering, background from He gas and sample cell, and thereafter the normalization with the incident X-ray spectrum, the structure factors $S(Q)$ were obtained. The X-ray scattering measurements of expanded 1-Se were performed along $T$ and $P$ indicated by red circles (see Fig. 1). The solid lines show the contours of constant DC conductivity $(\sigma)$ in expanded l-Se. The green line denotes the isochore of $d=3.4 \mathrm{~g} / \mathrm{cm}^{3}$. The analyses along the isochore give a distinctive response to a change of chain geometry in expanded l-Se near the SC-M transition.

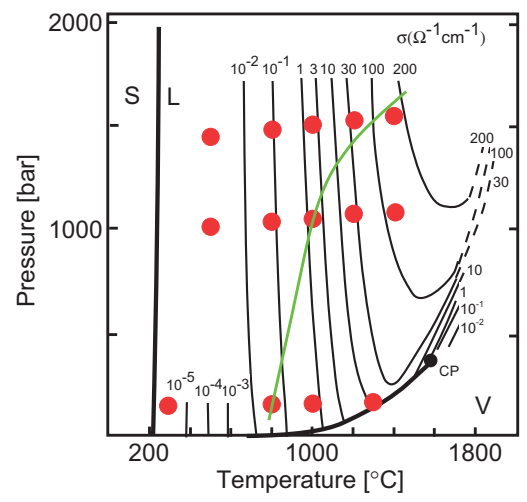

Figure 1. Pressure-temperature phase diagram[6,7] of selenium showing contours of constant DC electrical conductivity in the liquid and supercritical fluid. Green line shows the isochore of $d=3.4$ $\mathrm{g} / \mathrm{cm}^{3}$.
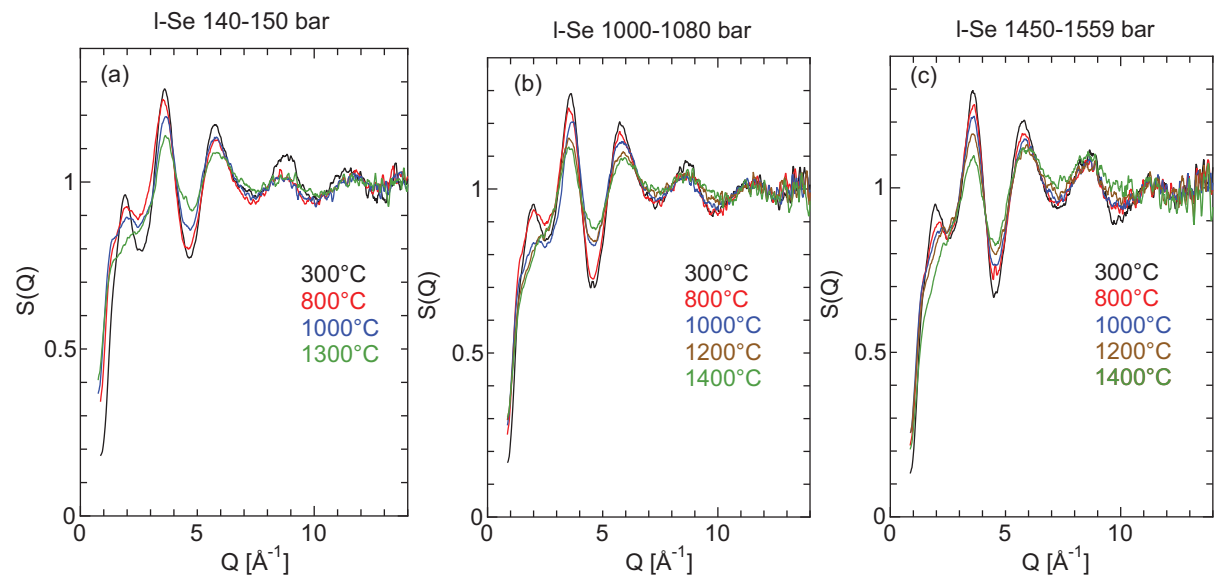

Figure 2. The temperature variations of the structure factor $\mathrm{S}(\mathrm{Q})$ for 1-Se under 140-150 bar (a), 10001080 bar (b), and 1450-1559 bar (c).

Fig. 2 shows temperature variations of the structure factor $S(Q)$ for l-Se under $140 \sim 150$ bar (a), $1000 \sim 1080$ bar (b) and $1450 \sim 1559$ bar (c).

The radial distribution functions $g(r)$ for l-Se along $d \sim 3.4 \mathrm{~g} / \mathrm{cm}^{3}$ at $800{ }^{\circ} \mathrm{C}(147 \mathrm{bar})$, $1000{ }^{\circ} \mathrm{C}$ (1052 bar), $1200{ }^{\circ} \mathrm{C}$ (1536 bar) and $1400{ }^{\circ} \mathrm{C}$ (1559 bar) are shown in Fig. 3. The peaks of $g(r)$ at $2.4 \AA$ give the bond lengths along a chain. The first peak exhibits a decrease in intensity at high temperature. The dip around $r \sim 3 \AA$ becomes less deep above $1100^{\circ} \mathrm{C}$ 
near the SC-M transition, which may be associated with a change of chain geometry (helical $\rightarrow$ zigzag) as discussed later.

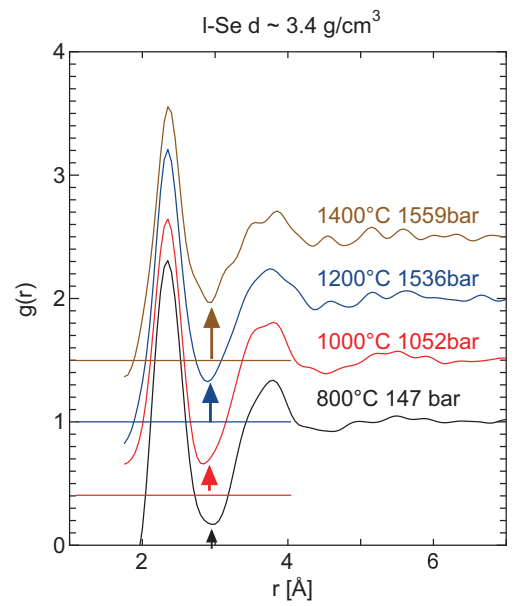

Figure 3. The pair distribution functions $g(r)$ of 1-Se along the isochore of $d \sim 3.4 \mathrm{~g} / \mathrm{cm}^{3}$. The arrows indicate the depth of the dip of $g(r)$ around $r \sim 3 \AA$.

The RMC simulation and VD void analyses have been applied to clarify the relationship between the packing of chains and interstitial voids in the intermediate scale. The void radius $r_{v}$ is given by that of the sphere circumscribing the DV tetrahedron. Fig. 4 (a) shows the size distribution of voids $P\left(r_{V}\right)$ in 1-Se along $d \sim 3.4 \mathrm{~g} / \mathrm{cm}^{3} . P\left(r_{V}\right)$ exhibits peaks or shoulders at $r_{V} \sim 2.8 \AA$ and $r_{V} \sim 2.3 \AA$ and additional peak around $r_{V} \sim 3.6 \AA$ as illustrated in Fig. 4(b). The peak at $r_{V} \sim 2.8 \AA$ gives a measure of the interstices between neighboring chains which is defined by the tetrahedron formed between three neighboring Se atoms along a chain and an atom belonging to the neighboring chains as illustrated in Fig. 5(a). As illustrated in Fig. 5(b) the peak at $r_{V} \sim 2.3 \AA$ is assigned to the radius estimated for the stacked zigzag chains driven by inter-chain coupling, which leads to the formation of metallic domain (M-domain)
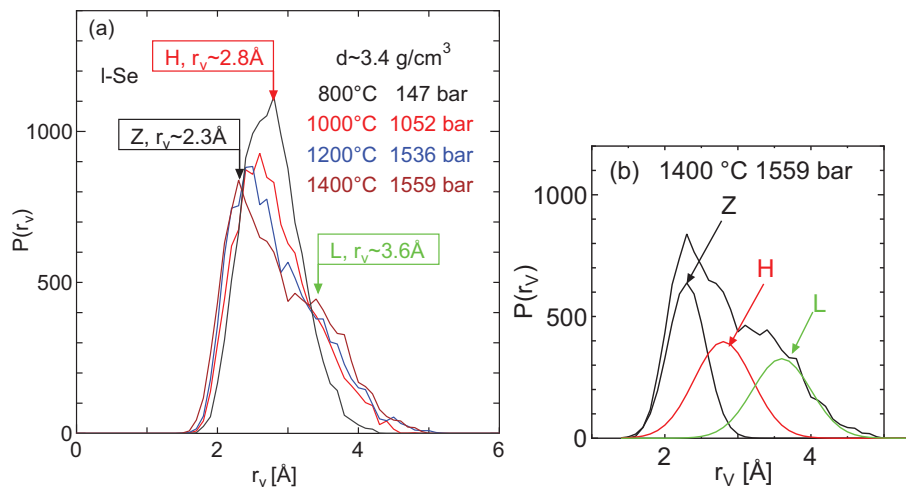

Figure 4. (a) The size distribution of void, $P\left(r_{V}\right)$ in the 1-Se along the isochore of $d \sim 3.4 \mathrm{~g} / \mathrm{cm}^{3}$. as a function of the void radius, $r_{V}$, of VD circusmsphere. (b) Gaussian curve fitting is shown in $1400{ }^{\circ} \mathrm{C}$ and 1559 bar. 


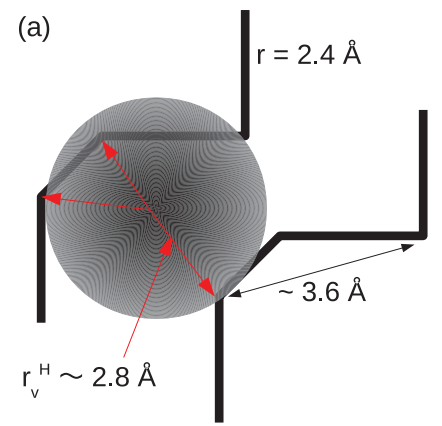

Radius of circumsphere between helical chains $r_{v}^{H} \sim 2.8 \AA$

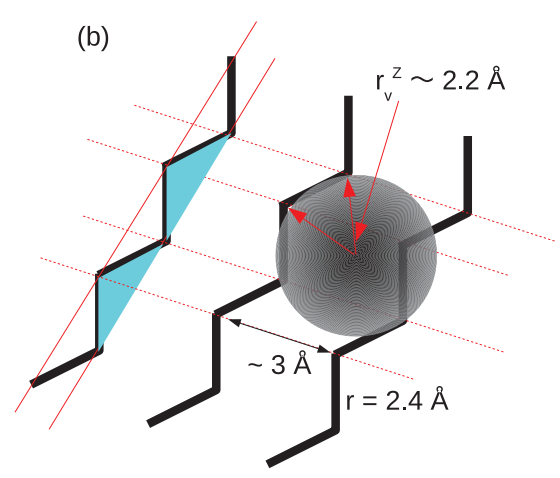

Radius of circumsphere between zigzag chain $r_{v}{ }^{z} \sim 2.2 \AA$

Figure 5. Schematic helical (a) and zigzag (b) chain configurations of expanded 1-Se around the void.

The geometrical requirement for stacked planar zigzag chain is compensated by empty space (L-voids, $r_{V} \sim 3.6 \AA$ ). The temperature variations of number fraction of voids $N$ with $r_{V} \sim 2.8,2.3$ and $3.6 \AA$ along $d \sim 3.4 \mathrm{~g} / \mathrm{cm}^{3}$ is shown in Fig. 6. The proportions for zigzag chains increase near the SC-M transition region $\left(\sim 1100^{\circ} \mathrm{C}\right)$. The shortening of chains, fluctuation of dihedral angles and branched chains with increasing temperature and pressure causes modification of helical chain to planar zigzag chain configurations. The distributions of helical $(\mathrm{H})$ and zigzag $(\mathrm{Z})$ chains are characterized by the distance to $n$th neighbor atom ( $n: 4 \sim 6$ ) along a chain. It is interesting to note that the distribution of distance assigned to $\mathrm{H}$ and $\mathrm{Z}$ give distinct peak as shown in Fig. 7, which suggests that chain geometry in expanded l-Se near the SC-M transition contains a stable planar zigzag conformation[8,9].

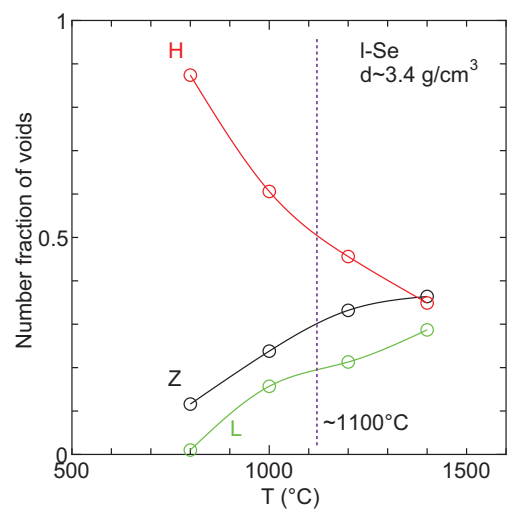

Figure 6. The tenperature variations of the number fraction of voids in expanded l-Se at $d \sim 3.4 \mathrm{~g} / \mathrm{cm}^{3}$ derived from the Gaussian fitting of the size distribution of voids, as shown in Fig.4(b).

Fig. 8 shows the temperature variations of number fractions, $N_{Z} / N_{H}$, for zigzag to helical chain configurations characterized by the distances to neighbor atoms $(n=4)$ along $\mathrm{H}$ and $\mathrm{Z}$ chains. The proportion of $\mathrm{Z}$ chains with metallic character increases near the SC-M transition, which is consistent with the results in Fig. 6. 

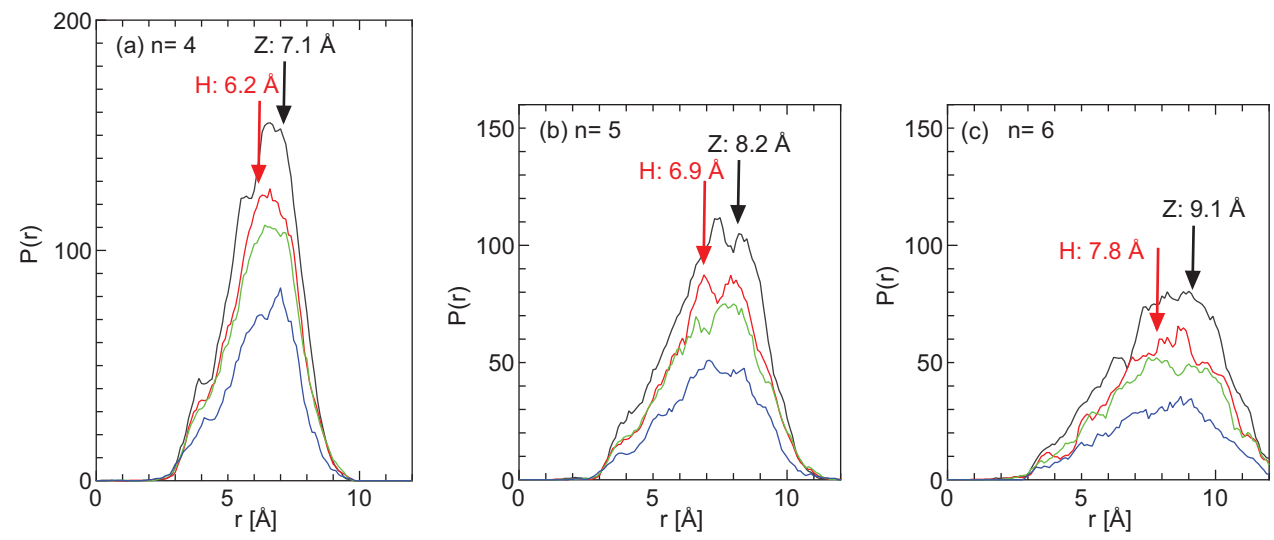

Figure 7. Distribution of atomic distances $P(r)$ along a chain: black curve indicate $P(r)$ for $800^{\circ} \mathrm{C}$ (147bar), red for $1000^{\circ} \mathrm{C}(1052 \mathrm{bar})$, green for $1200^{\circ} \mathrm{C}$ (1536bar) and blue for $1400^{\circ} \mathrm{C}$ (1559bar). (a)distribution of $n=4$, (b) $n=5$, (c) $n=6$. Red arrows indicate the position assigned to helical chain and black arrow position assigned to zigzag chain.

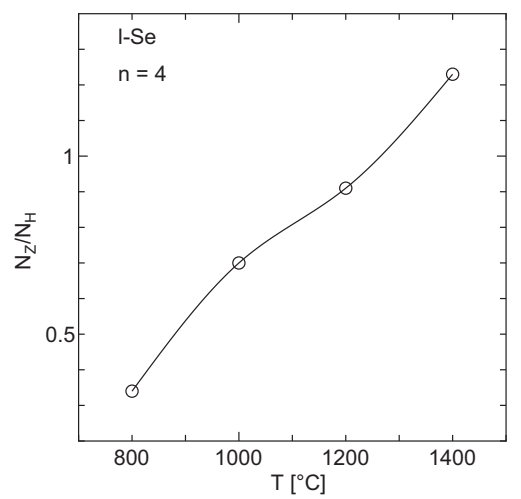

Figure 8. The temperature variatons of number fraction $N_{Z} / N_{H}$ for $\mathrm{Z}$ and $\mathrm{H}$ chain configuratins characterized by distance to 4 th neighbor atom along a chain as shown in Fig. 7(a).

\section{Conclusion}

X-ray scattering measurements were performed on the expanded liquid Se near the SC-M transition along the isochore of $d=3.4 \mathrm{~g} / \mathrm{cm}^{3}$. RMC and VD void analyses were applied to clarify the modification of chain geometries in intermediate scale in expanded liquid Se near the SC-M transition region.

The helical chains are modified to the stacked planar zigzag chain which leads to the formation of metallic domains. The distributions of $\mathrm{H}$ and $\mathrm{Z}$ chains are characterized by the distribution of void sizes supported by chain segments and the distances to neighbor atoms along a chain. It is found that near the SC-M transition region number fraction of $N_{Z} / N_{H}$ for $\mathrm{Z}$ to $\mathrm{H}$ chain segments increases and is suggestive of micro-segregation [10] of helical and zigzag chain segments. 


\section{References}

[1] R. L. McGreevy, J. Phys.: Condens. Matter 13, R877 (2001)

[2] S. L. Chan and S. R. Elliot, Phys. Rev. B43, 4423 (1991)

[3] P.A. Madden and M. Wilson, Phys. Rev. Lett. 80, 532 (1998)

[4] K. Tamura and M. Inui, J. Phys.: Condens. Matter 13, R337 (2001)

[5] K. Tamura and S. Hosokawa, Ber. Bunsenges. Phys. Chem. 96, 681 (1992)

[6] H. Hoshino, R. W. Schmutzler and F. Hensel, Ber. Bunsenges. Phys. Chem. 80, 27 (1976)

[7] F. Hensel and W. W. Warren, Jr., Fluid Metals (Princeton Univ. Press, Princeton, 1999) chap. 5

[8] A. Ikawa and H. Fukutome, J. Phys. Soc. Jpn. 59, 1002 (1990)

[9] K. Maruyama, H. Endo, H. Hoshino, Y. Kajihara, M. Nakada and S. Sato, J. Phys.: Condens. Matter 22, 455103 (2010)

[10] H. Kajikawa, S. Takahashi, M. Iwakoshi, T. Hoshino and M.Yao, J. Phys. Soc. Jpn. 76, 014604 (2007) 